\title{
Improvement Strategies for the Service Quality of Hospitals
}

\author{
Jin Yang \\ School of Management and Economics \\ University of Electronic Science and Technology of \\ China, Chengdu 611731, China \\ dr.jinyang@163.com
}

\section{Xu Chen*}

School of Management and Economics

University of Electronic Science and Technology of

China, Chengdu 611731, China

xchenxchen@263.net

* Corresponding author

\author{
Ze Sun \\ School of Management and Economics \\ University of Electronic Science and Technology of \\ China, Chengdu 611731, China \\ sunzephy@gmail.com
}

\begin{abstract}
More and more public attentions have been paid to hospital service quality nowadays. Improving the quality of hospital service can ease the current tension in the doctorpatient relationship, as well as enhance the hospital's competitiveness. The paper is aimed to propose a comprehensive plan to improve the service quality of hospitals. We analyzed the reasons for low service quality of hospitals in detail, and put forward a series of appropriate strategies. Especially, we emphasized the importance of the application of information tools and the improvement of the satisfaction of medical staff.
\end{abstract}

Keywords- service quality; hospital; patient satisfaction; medical staff

\section{INTRODUCTION}

Hospital is a special kind of service organization, which has the attribute of the general service industry. At the same time, it has some characteristics which differ from the features of other service industries. For a long time, hospitals are focusing on improving their medical technologies and helping to relieve pains of patients during this process, what hospitals concern most is the quality of medical care. However, with the progress of medical technologies and the changes of market environment in recent years, more and more hospitals begin to pay more attentions to the improvement of service quality.

For hospitals, with the deepening of the health system reforms in China, the government has increasingly intensified the policy efforts and expanded financial investment on infrastructure construction of health care, leading to a significant increase in the level of the construction of grassroots medical institutions.

In addition, in recent years, the policy on establishing social medical institutions has been much eased, and the opening of medical market is already on track. Furthermore, medical institutions such as private hospitals, joint venture cooperation hospitals, and wholly foreignowned hospitals have been developed rapidly. As a result, the distribution of medical resources becomes more reasonable and more uniform, and the gap between medical technologies of different types of hospitals is narrowed, thus making patients have more available choices when they accept conventional disease diagnosis and treatment. These changes also have brought the homogeneity of hospital services as well as the increased competition between hospitals. In such a competitive environment, taking differentiation competitive strategies and providing high quality services have become efficient methods for hospitals to improve their competitiveness and economic benefits.

For patients, with higher per capita income and higher living standards in China, people's requirements for better hospital services are generally observed, and their demands for multiple levels and diversity of medical services have become more obvious. When patients choose hospitals, the quality of hospital services has become secondary key factor to consider besides the level of medical technology.

Considering from both the current situation of competition of hospital industries and the demands of patients, the quality of hospital service is of great importance. Most hospitals have realized the significance of medical service, and they have also taken some efforts to improve the quality, however, few of them success.

A serious mismatch is caused by the imbalance between low service quality and demand of patients, which leads to low patient satisfaction strained relationship between doctors and patients, and frequent medical disputes. As a result, it is imperative to improve the quality of hospital services in scientific and effective ways.

\section{LITERATURE REVIEW}

The quality of service in hospitals involves many factors. A large number of researches was conducted by scholars to improve the service quality of hospitals from different points. Taylor, S. A. (1994) considered the development of the service quality in hospitals as a profound marketing strategy. L.X. Li. (1997) empirically 
explores the relationship between hospital quality management and service quality performance in US community hospitals by using a path analytic model. This research revealed strong correlations among hospital service quality performance, the analys is of service process and the workforce development. Miao Zhimin and Zhao Shihua (2001) from the perspective of logistics services put forward the use of the socialization and enterprization of logistics services to improve service levels. Raduan Che Rose, Jegak Uli, Mohani Abdul, Kim Looi Ng, (2004) found that the cost factor is insignificant for hospital service quality prediction. Zhu Zongming and Zhu Shaoming (2004) from the perspective of communication between doctors and patients, put forward that "zero defect" communication is an effective way to improve the quality of hospital services. Feng Wei (2005) proposed to improve the hospital service quality and service efficiency through the hospital treatment process reengineering. Zhou Wuying and Liu Yongqiang (2006) from the perspective of strengthening the management of complaints to improve the hospital service quality. Yang Anyu et al. Jiunn-I Shieh, Hsin-Hung $\mathrm{Wu}$ and Kuan-Kai Huang (2010) firstly conducted the survey based on SERVQUAL model to identify seven major criteria for service quality of the hospital from patients' or their families' viewpoints. Yang Anyu (2010) put forward that PDCA cycle is applied to implement the quality improvement of hospital outpatient service. Tina (2013) said using the control chart method to monitor the change trend of the hospital service quality evaluation index, and find the abnormal fluctuation in time, and make the corresponding improvement measures. Timothy R. Furey (1991) emphasized importance of the information technology to a sophisticated statistical system for measuring service quality.

There is a lot of relevant literatures. Hospital service quality is different from medical quality. Medical quality refers to the rehabilitation effect of patients with the disease, the virtuosity of medical personnel, the advanced technology of medical equip ment, and the high standard of medical and health. And the service quality of hospitals emphasis on patient's experience in the treatment process, covering hospital medical technology, medical staff service attitude, hospital environment and facilities, all aspects patients might be exposed to. On the whole, on the one hand, the existing research results focus on medical quality management and improvement, on the other hand, these studies mostly focus promotion strategy of hospital service only from single dimension, which is incomplete.

\section{ANALYSIS OF THE REST RICTING FACT ORS OF HOSPIT AL SERVICE QUALITY}

\section{A. The enthusiasm of the medical staff is not high.}

Currently, when hospitals want to improve service quality and improve patient satisfaction, most of them simply consider how to serve patients better, ignoring satisfaction of the internal employee, which largely limits the enthusiasm for the medical staff to service patients. The main reasons leading to the low enthusiasm of health care workers are the following several aspects.

The first is inadequate staffing, unreasonable scheduling, high work intensity, and long-term high-load work. The second is the low level income, which does not match the work intensity and pressure. The third is lack of the support from administrative, logistics departments. The Forth aspect is that the performance evaluation system is not scientific, the existing evaluation system focused primarily on the assessment of the economic indicators, without closely linked with the service quality and levels, failing to achieve its proper incentives.

\section{B. Lagging level of informatization}

Applications of information technology can improve efficiency, many domestic hospitals have carried out a certain degree informatization, and the informatization initiatives are mostly to digitize traditional medical processes, without make full use of informatization to optimize the treatment process. The information construction of hospitals is lagging behind, the information of each department cannot be shared in time, resulting in the repeated labor and low work efficiency of the medical staff, bring patients the unnecessary waiting and working.

\section{Lack of service enhancement mechanism}

It is not uncommon for domestic hospitals to hold activities such as "the year of enhancing services", "service month". However, these activities are usually with little success. Hospitals invested a lot of manpower and resources to carry out these activities, but the quality of service had a little improvement, but after the end of these activities the level of service quality remains stagnant. The reason lies in the lack of long-term effectively operating mechanism to improve the service quality. It is reflected in: lack of scientific planning system before the activity; lack of effective supervision during the activity, the measures cannot be implemented correctly; after the activity, there is no continuous improvement based on the service status and feedback.

\section{HOSPIT AL SERVICE QUALITY IMPROVEMENT STRATEGY}

\section{A. Improve employee satisfaction}

Internal employee satisfaction has a direct impact on work enthusiasm of medical personnel when they provide services to patients, only when medical staff themselves are satisfied, they can service patients in good working state. According to a study by Harvard University, the satisfaction of customer increases 5 percentage when the satisfaction of employees improves per 3 percentage points. There are many factors that affect the satisfaction of employees, the following measures can be taken in the current situation of our hospitals.

(1) Strengthen the professionalization of administration in the hospital and the socialization of logistics. Connect the evaluation of health care workers with the performance evaluation of administrative and logistics, truly realize that administration and logistics service clinic. (2) Reform the remuneration structure, improve the assessment mechanism. Hospitals should establish professional salary management team and reform salary structure, and should develop a scientific, objective, transparent, and fair assessment standard, to provide doctors and nurses both competitive and incentive Salary. (3) Configure the personnel reasonably. On the one hand, carry on quantitative analysis of the existing 
staffing, business volume and intensity of work, add clinical human properly. On the other hand, according to the business volume, achieve the dynamic, flexible management with different department and different period of time, flexibly and reasonably use limited medical personnel.

\section{B. Improve service awareness}

Awareness of service refers to the desires and sense to provide enthusiastic, considerate and active service when the entire staff contact with all corporate stakeholders, which is a kind of concept and desire to service consciously and actively. The true sense of service should be the psychological orientation to provide service to customer from the heart totally, without considering about the rules and regulations, evaluation standard and the improvement of salary. The service that is dominated by this consciousness is the real service. Service consciousness is a kind of instinct and habit of service personnel, which can be formed from training and education. The hospital can carry out service training course for all employees to improve the service consciousness of the staff.

\section{Make clear the standards of quality service}

By the two measures above, employees have a good working state and good intentions of customer service. At this time, the problem hospitals may face is that the staff feels powerless, that means, they want to serve their customers, but do not know what kind of service is good service and how to provide customers good service. In order to solve this problem, the hos pital haves to clear the standard of quality service, to clear specification and refine the service standards in an institutional way. Make the employee service manual, and organize the staff to study the service manual deeply and systematically.

\section{Full use of information technology}

Application of information technology is essential for improving the efficiency of medical personnel and the patient experience. Hospital informatization should not be just for the digitization of traditional treatment process only, but also the treatment process reengineering with patient centered based on networking, the Internet, mobile Internet, big data, social networks, mobile payment and other technology. Through process reengineering, delete the unnecessary steps, simplify the necessary steps, in order to reduce patient waiting times and shorten patient waiting time. In addition, the information technology can also help the hospital to achieve the service which cannot achieve in the past, further expand the service contents and improve the patient experience. For example, prediagnosis can be carry out before the patients reach the hospital, achieving real-time communication between the medical staff and the patient and the tracking services after the patient left the hospital.

\section{E. Establish and improve the service promotion mechanism}

It is not easy to enhance the quality of service. Hospitals should establish and improve the mechanis $m$ to improve the service, improve the normalization of the service work. Arrange the particular person to be responsible for the work to improve service quality. and arrange a professional team to promote the implementation. Service improvement is endless; Any detail improved might bring a better experience to patients. Hospitals should encourage service innovation, and encourage all employees to think about the direction and the measure summarized from the practical experience by the medical staff who directly contact with patients.

\section{THE SUPPORT TO IMPROVE THE SERVICE QUALIT Y} OF HOSPIT AL

\section{A. Organizational support}

The hospital should establish a dedicated service management department, which is different from the traditional hospital quality management, it does not focus on the quality of medical attention, but the patient's experience. Service management department is responsible for the management of the hospital service quality, the handling of service complaints, the service process optimization, and formulation to lift the service level, implementation of improvement measures, the service consciousness of the hospital staff, service standard training and other affairs. The service management department should be independent from other departments, and hospitals have to identify responsibilities and privileges belong to the department and other departments in terms of enhancing the service to ensure that service improvement activities can be carried out smoothly.

\section{B. Manpower Support}

Service quality improvement involves many aspects. Many new requirements are brought to the human resources of the hospital. Most of the hospital's existing management personnel are promoted from the grassroots medical staff. Although these people have rich medical knowledge and clinical experience, they generally lack of management knowledge and management skills. These people have the rich medical knowledge and clinical experience, but lack of management knowledge and management ability generally. On the one hand, the hos pital should broaden the scope of recruiting, not limit to the professional medical or health management talents, just base on the needs to introduce the service management, service process design and optimization, information system development and other professionals. On the other hand, the hospital should give the existing management personnel the training of service management knowledge and skills, innovative their service concept, enhance their service innovation awareness.

\section{Culture Support}

Organizational culture is the value concept and the code of conduct recognized by all the staff of, a hospital should build a organization culture system that consis ts of four types of sub-culture system, service culture, efficiency culture, team culture and learning culture. Among them, the connotation of the service culture is to make sense of service deep into each internal stuff's heart, let they treat service as a natural expression rather than the task; The connotation of efficiency cultural refers to stressing both quality and speed, not just speed in the process of hospital services, providing patients faster and better service; The connotation of team culture refers the solid teamwork spirit formed among the hospital departments or colleagues 
within a department. No excuse, no neglect, service every aspect of patient together; the connotation of learning culture is to transform learning into organizational behavior, improve staff service level and service ability with continuous learning, give hospitals sustained competitive advantage with learning from the successful experience of peers and learning and continuous innovation of hos pital services.

\section{CONCLUSIONS}

Firstly, this paper demonstrated the urgency to enhance the quality of hospital service by analyzing two aspects in detail: competition of hospitals and demand of patients. Secondly, we discussed the main factors that restricts the improving of hospital service quality in China. Furthermore, we put forward appropriate strategies to obtain better service quality in hospitals. Finally, practical several measurements to implement the strategies mentioned above was pointed out from the three aspects: organization, human resource, and culture. The research of this paper is of guiding significance for helping to improve the service quality and the sustainable competitive advantage for hos pitals.

\section{ACKNOWLEDGMENTS}

This research is partially supported by National Natural Science Foundation of China (No. 71272128, 71432003), Program for New Century Excellent Talents in University (No. NCET-12-0087), and Specialized Research Fund for the Doctoral Program of Higher Education (No. 20130185110006).

\section{REFERENCES}

[1] Taylor, Steven A, "Distinguishing service quality from patient satisfaction in developing health care market ing strategies" Journal of Healthcare Management, vol 39, pp.221-236,1973.
[2] L.X. Li, "Relationships between determinants of hospital quality management and service quality performance - a path analytic model" Omega, vol 25, pp. 535-545, 1997, doi:10.1016/S03050483(97)00017-0.

[3] Miao Zhimin and Zhao Shihua, "Changes in hospital logistics management mechanism, improve service quality" Chinese Journal of Hospital Administration, vol 16, pp.468-469, 2000.

[4] Raduan Che Rose, Jegak Uli, Mohani Abdul, Kim Looi Ng, "Hospital service quality: a managerial challenge" International Journal of Health Care Quality Assurance, Vol. 17, pp.146 - 159, 2004, doi: 10.1108/09526860410532784

[5] Zhu Zongming, Zhu Shaoming, "Implement zero defect communication between the doct or and the patient to enhance the quality of hospital services " Chinese Health Quality Management, vol 11, pp. 24-26, 2004.

[6] Feng Wei, "Process reengineering: scientific methods for improving the quality and efficiency of medical services - a summary of the Symposium on the process reengineering in China and the United St ates" Chinese hospital, vol 9, pp. 75-77, 2005.

[7] Zhou Wuying, Liu Yongqiang, "Strengthen hospital complaints management to improve the quality of medical services" J Chinese hospital, vol 11, pp. 52-53, 2007.

[8] Shieh, Jiunn-I., Hsin-Hung Wu, and Kuan-Kai Huang, "A DEMATEL method in identifying key success factors of hospital service quality" Knowledge-Based Systems, vol 23, pp. 277-282, April 2010, doi:10.1016/j.knosys.2010.01.013

[9] Shen Tianjie, Yang Anyu, Wu Lili, "PDCA circulation management mode to promote the quality of outpatient medical services continued to improve" Journal of Traditional Chinese Medicine Management, vol 18, pp. 536-537, 2010.

[10] J Ding Na, Yao Yao, Li Miao, "I Hospital service quality continuous improvement based on control chart" Chinese Hospital Management, vol 33, pp. 76-79, 2013.

[11] Furey, Timothy R, "How information power can improve service quality" Planning Review, vol 19, pp. 24-26, 1991, doi: http://dx.doi.org/10.1108/eb054325. 